patients with pulmonary complications of rheumatoid arthritis (RA) and Sjögren's syndrome (SS) showed the greatest prevalence of induced BALT. This tissue differed from classical BALT because it resembled ectopic lymphoid follicles and was found throughout the airways.

The authors examined lung biopsy specimens from 36 patients with one or more interstitial lung diseases: idiopathic interstitial pneumonia $(n=10)$; hypersensitivity pneumonitis $(n=6)$; idiopathic pulmonary fibrosis $(n=7)$; chronic infectious disease ( $n=4)$; cancer $(n=4)$; and RA $(n=9)$ or SS $(n=3)$ with pulmonary complications.

Patients with pulmonary manifestations of RA or SS consistently had the largest and most numerous areas of induced BALT (present in eight of nine RA patients and all three SS patients), although phenotypes varied. By contrast, BALT was present in only two patients with idiopathic interstitial pneumonia and two with hypersensitivity pneumonitis, which suggested that BALT is not induced merely by pulmonary inflammation.

In patients with RA, increased size, number and organization of BALT patches correlated with elevated local expression of cytokines, costimulatory molecules and enzymes involved in RA pathogenesis. The authors speculate that the development of induced BALT in patients with RA or SS is triggered by autoantigen exposure.

Original article Rangel-Moreno J et al. (2006) Inducible bronchus-associated lymphoid tissue (iBALT) in patients with pulmonary complications of rheumatoid arthritis. J Clin Invest 116: 3183-3194

\section{Regulatory T cells and interleukin 17 in autoimmune disease}

Much interest has been raised in harnessing the immunomodulatory effects of regulatory T cells $\left(T_{R E G} s\right)$ to treat autoimmune disorders. There is evidence that $T_{R E G S}$ stimulate the production of interleukin 17 (IL-17), which is an important mediator of tissue inflammation in inflammatory disease models. Transfer of $T_{R E G} S$ might not, therefore, be a suitable therapeutic approach for IL-17-mediated diseases. Lohr et al. explored the roles of cytokines and $T_{R E G} s$ in a mouse model of autoimmune inflammatory disease, in which T-cells that recognized an ovalbumin fragment were introduced into mice that systemically secreted ovalbumin.
The researchers established that IL-17producing $T$ cells were responsible for the tissue-dependent inflammatory response seen in this model; the skin inflammation could be abolished by treatment with an anti-IL-17 antibody. Disease symptoms were exacerbated in the absence of a type $1 \mathrm{~T}$ helper $\left(T_{H} 1\right)$ cell response - when mice were injected with T cells from $I_{\text {fng }}{ }^{--}$or Tbx21-- knockout mice (which lack a $T_{H}{ }^{1-s p e c i f i c ~ t r a n s c r i p t i o n ~ f a c t o r), ~ s e v e r e ~}$ skin lesions appeared. Although $\mathrm{T}_{\mathrm{REG}} \mathrm{s}$ promote IL-17 production in vitro, they prevented disease in vivo when introduced early in the disease course but were ineffective against established disease.

The authors state that $T_{R E G} S$ can prevent autoimmune disease by blocking the activation and expansion of effector cells, but might be of little benefit in the treatment of established conditions. $T_{R E G} s$ might also be ineffective in treating IL-17-mediated inflammation, because of their role in promoting $\mathrm{IL}-17$ production.

Original article Lohr J et al. (2006) Role of IL-17 and regulatory $\mathrm{T}$ lymphocytes in a systemic autoimmune disease. J Exp Med 203: 2785-2791

\section{HLA-DRB1 SE genotype predicts severe erosive disease in some patients with RA}

A study has shown that, in patients with rheumatoid arthritis (RA), the value of genotyping for HLA-DRB1 alleles that include a shared epitope (SE) sequence as a predictor of severe erosive disease varies according to individuals' other risk factors for severe disease. Although the SE was known to predict severe erosive disease, its additional value with respect to other predictors of severe disease was unknown.

The study included 154 white women with RA, followed for a mean of 12 years. Severity of erosive disease was measured by hand and wrist radiographs; each patient was genotyped, and demographic, clinical and immunologic risk factors for RA were considered.

The study showed that absence of the SE lowered the probability of severe erosive disease by $\geq 50 \%$, regardless of other risk factors; however, presence of the SE did not increase the risk of severe erosive disease when other risk factors were present. Among rheumatoidfactor-negative patients, SE positivity increased the risk of severe disease. 